\title{
Adenomatoid tumor of kidney - a rare histological entity: case report with review of literature
}

\begin{abstract}
Adenomatoid tumors of Genito-urinary Tract are rare neoplasias, with benign behaviour, and usually are diagnosed incidentally. Adenomatoid tumor of Kidney is even rarer. We report a case of adenomatoid tumor of left kidney in a 53-year old woman who sought evaluation because of vague abdominal pain for one year. During the investigation, aexophytic complex cystic lesion $(10 \mathrm{x} 8 \mathrm{~cm})$ in the upper pole of left kidney was identified on ultra sonography. The patient underwent left radical nephrectomy and histo pathological diagnosis was adenomatoid tumor of kidney. The patient is on follow up for last $3 \mathrm{yrs}$ and is doing well.
\end{abstract}

Keywords: adenomatoid tumor, rare renal tumors, immunohistochemistry

\author{
Volume 2 Issue 2 - 2015
}

\begin{abstract}
Sasanka Kumar Barua, Dhekial Phukon P, Sharma Devanga, Bagchi Puskal, Rajeev TP Department of Urology, Gauhati Medical College and Hospital, India
\end{abstract}

Correspondence: Sasanka Kumar barua, Gauhati Medical College, Guwahati, Assam, India, 781032, Tel +919864096583, Email sasankagmch@gmail.com

Received: January 03, 2015 | Published: March 19, 2015

\section{Introduction}

Adenomatoid tumors are benign neoplasm of mesothelial origin, frequently afflicting the genital organs. ${ }^{1}$ They usually follow a benign course and are usually detected incidentally on imaging. Renal adenomatoid tumors are extremely rare and there is paucity of report of such cases in the literature. We report here a case of Adenomatoid tumor of kidney in a $53 \mathrm{yrs}$ old female.

\section{Case presentation}

A 53years old obese woman presented with vague abdominal pain of one year duration. Physical examination was unremarkable. Ultra sonography of the whole abdomen detected an exophytic complex cystic lesion measuring about $10 \mathrm{x} 8 \mathrm{~cm}$ in upper pole of left kidney (Figure 1). MDCT whole abdomen showed $9.4 \times 7.7 \times 7.5 \mathrm{~cm}$ sized multiloculated cystic lesion with enhancing septae and calcifications with solid nodular areas (Figure 2). The patient was diagnosed as Stage II left Renal Cell Carcinoma and subjected to Radical Nephrectomy. Adrenal was also removed as the tumor was in upper pole. On gross examination the tumour was multi nodular. Cut surface shows a spongy mass measuring $11 \times 8.5 \times 5 \mathrm{~cm}$, filled with yellowish serous fluid in the upper pole. On microscopic examination, the tumour was composed of variably sized tubules and fenestrated channels lined by bland cuboidal cells to epithelioid cells. No mitosis, cytological atypia, or nuclear pleomorphism was seen. Remaining kidney showed patchy chronic pyelonephritis with glomerulosclerosis and scarring. The adrenal showed normal histological appearance. Histopathology of the tumor was suggestive of Adenomatoid tumor of kidney (Figure $3)$. The tumor was positive for vimentin on immunohistochemistry.

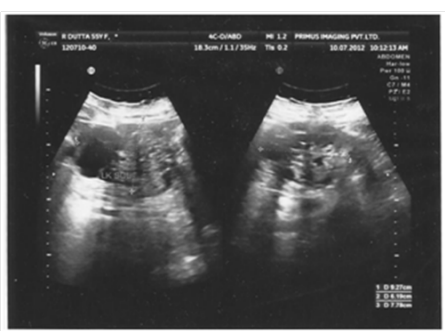

Figure I Exophytic complex cystic lesion $(10 \times 8 \mathrm{~cm})$ in upper pole of left kidney.

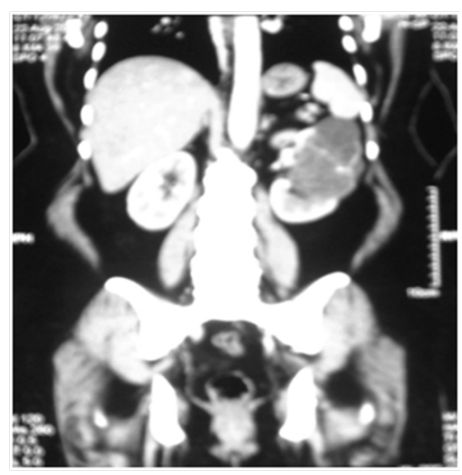

Figure 2 Multiloculated cystic lesion with enhancing septae and calcifications with solid nodular areas.

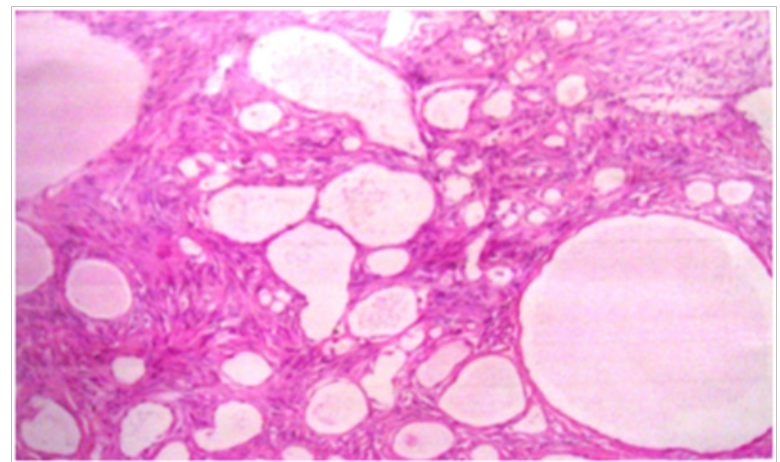

Figure 3 Tumour composed of variably sized tubules and fenestrated channels lined by bland cuboidal cells to epithelioid cells.

\section{Discussion}

Adenomatoid tumors are rare benign neoplasm of mesothelial origin. Frequently afflicts the genital organs, but rarely reported in extra-genital sites. ${ }^{2}$ Testis, uterus, ovary, adrenal glands are frequent sites in urogenital tracts. There are 8 cases describing adenomatoid tumor of adrenal gland, occurring mostly in males, involving more frequently the left adrenal gland. ${ }^{3}$ Immunohistochemistry is often found to be positive for vimentin which is also seen in our case. ${ }^{4}$ But to our knowledge no case of adenomatoid tumor of kidney has been reported in the literature so far. Apparently, there are no 
specific characteristics that enable us to radio logically distinguish adenomatoid tumors from other renal tumors. There are no reports in the literature of lesion recurrence following complete surgical removal, and surgical extirpation is considered curative. ${ }^{5}$

\section{Acknowledgements}

None.

\section{Conflict of interest}

The author declares no conflict of interest.

\section{References}

1. Canzonieri V, Volpe R, Gloghini A, et al. Mixed renal tumor with carcinomatous and fibroleiomyomatous components, associated with angiomyolipoma in the same kidney. Pathol Res Pract. 1993;189(9):951956.
2. Glatz K, Wegmann W. Papillary adenomatoid tumour of the adrenal gland. Histopathology. 2000;37(4):376-377.

3. Raaf HN, Grant LD, Santoscoy C, et al. Adenomatoidtumor of the adrenal gland: a report of four new cases and a review of the literature. Mod Pathol. 1996;9(11):1046-1051.

4. Schwartz EJ, Longacre TA. Adenomatoid tumors of the female and male genital tracts express WT1. Int J Gynecol Pathol. 2004;23(2):123-128.

5. El-Daly H, Rao P, Palazzo F, et al. A rare entity of an unusual site: Adenomatoid tumour of the adrenal gland:A case report and review of the literature. Patholog Res Int. 2010:702472. 